\title{
Stage 0 Intrahepatic Bile Duct Cancer AJCC v8
}

National Cancer Institute

\section{Source}

National Cancer Institute. Stage O Intrahepatic Bile Duct Cancer A/CC v8. NCI Thesaurus. Code C134609.

Stage 0 includes: T is, NO, MO. T is: Carcinoma in situ (intraductal tumor). N0: No regional lymph node metastasis. M0: No distant metastasis. (AJCC 8th ed.) 